\title{
Primary leiomyosarcoma of the liver: Two new cases and a systematic review
}

\author{
Francesco Esposito ${ }^{1}$, Chetana Lim², Laurence Baranes ${ }^{3}$, Chady Salloum ${ }^{4}$, \\ Cyrille Feray ${ }^{4}$, Julien Calderaro ${ }^{5}$, and Daniel Azoulay ${ }^{4,6}$
}

\begin{abstract}
${ }^{1}$ Department of General Surgery, Meaux Hospital, Meaux, ${ }^{2}$ Department of Hepatobiliary and Pancreatic Surgery and Liver Transplantation, Pitié-Salpêtrière Hospital, Paris, ${ }^{3}$ Department of Radiology, Henri Mondor Hospital, Créteil, ${ }^{4}$ Department of Hepatobiliary and Pancreatic Surgery and Liver Transplantation, Paul Brousse Hospital, Villejuif, ${ }^{5}$ Department of Pathology, Henri Mondor Hospital, Créteil, France, ${ }^{6}$ Department of Hepatobiliary and Pancreatic Surgery and Transplantation, Sheba Medical Center, Faculty of Medicine, Tel Aviv University, Tel Aviv, Israel
\end{abstract}

\begin{abstract}
Primary hepatic leiomyosarcoma $(\mathrm{PHL})$ is a rare malignant tumor, which originates from smooth muscles. Clinical presentation and imaging features are non-specific and can mimick the most frequent primary liver tumors namely hepatocellular carcinoma and intrahepatic cholangiocarcinoma. We report here two cases of PHL including one from the portal vein. The literature was searched for studies reporting cases of PHL reported from 2011 and 2019. The two patients were operated with R0 resection. Diagnosis of PHL was confirmed by histopathological and immunohistochemical examinations. Surgery remains the mainstay of the management of PHL. RO resection is the main prognostic factor. Our literature search identified 16 additional cases from 12 reports. Preoperative diagnosis of PHL needs a high degree of suspicion due to atypical clinical presentation and non-specific imaging features. Surgery is the mainstay of the management of PHL. R0 resection is the main prognostic factor. (Ann Hepatobiliary Pancreat Surg 2020;24:63-67)
\end{abstract}

Key Words: Leiomyosarcoma; Liver; Primary; Hepatectomy; Portal vein

\section{INTRODUCTION}

Primary hepatic leiomyosarcomas (PHL) include 6-16\% of the primary hepatic sarcomas which in turn represent $0.2-2 \%$ of primary hepatic cancers. ${ }^{1}$ Primary hepatic leiomyosarcoma (PHL) is a rare malignant tumor, which originates from smooth muscles. Clinical presentation and imaging features are non-specific and can mimick the most frequent primary liver tumors namely hepatocellular carcinoma and intrahepatic cholangiocarcinoma. This tumor has aggressive metastatic potential and is usually diagnosed in a locally advanced or metastatic disease. Radical R0 hepatectomy remains the only curative treatment of PHL, but the large majority of unoperated patients had technically non-resectable disease and/or extra-hepatic metastases. We report here two cases of PHL including one arising from the portal vein to update the nosology, incidence, diag- nosis and management of this rare disease. The cases reported here comply with the CARE guidelines checklist for case reports. ${ }^{2}$

\section{CASE}

\section{Case 1}

A left liver mass was incidentally discovered during cardiac ultrasonography in an asymptomatic 78-year old male patient. Abdominal computed tomography (CT) showed a large tumor (57 $\mathrm{mm}$ in diameter), well defined, heterogeneous and spontaneously hypodense (Fig. 1A). Contrast imaging showed mild and mostly peripheral wash-in and no wash-out. The whole left portal vein system was enlarged by a tumor thrombus (TT) enhanced on arterial phase. On magnetic resonance imaging (MRI), the tumor was heterogeneous and hypointense on T1-weighted im-

Received: April 30, 2019; Revised: May 5, 2019; Accepted: May 9, 2019

Corresponding author: Daniel Azoulay

Department of Hepatobiliary and Pancreatic Surgery and Transplantation, Sheba Medical Center, Faculty of Medicine, Tel Aviv University, Israel. E-mail: daniel.azoulay@sheba.health.gov.il

Copyright (C) 2020 by The Korean Association of Hepato-Biliary-Pancreatic Surgery

This is an Open Access article distributed under the terms of the Creative Commons Attribution Non-Commercial License (http://creativecommons.org/ licenses/by-nc/4.0) which permits unrestricted non-commercial use, distribution, and reproduction in any medium, provided the original work is properly cited. Annals of Hepato-Biliary-Pancreatic Surgery • pISSN: 2508-5778 - eISSN: 2508-5859 

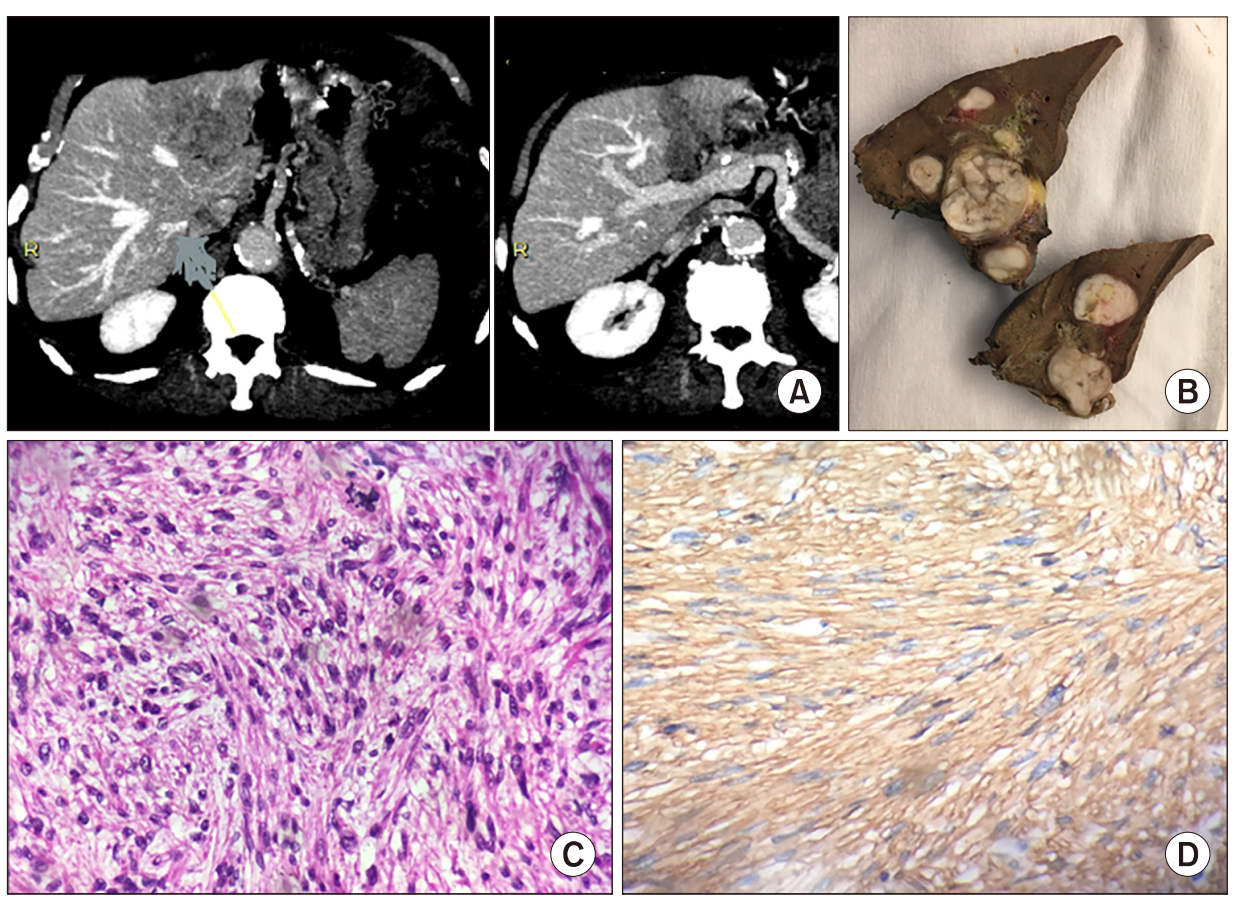

Fig. 1. Leiomyosarcoma of the left portal vein. (A) Computed tomography. (B) Resected specimen. (C) Microscopic examination shows a tumor composed of atypical spindle cells with a fascicular growth pattern. Atypical mitoses were observed. (D) Immunohistochemical staining against alpha-smooth muscle actin was strongly positive. ages and hyperintense on T2-weighted images without encapsulation. At arterial and portal phase, the TT was discretely hypervascular. The non-tumor liver parenchyma was normal on cross imaging. Thorax and pelvis imaging as well as upper gastrointestinal and recto-colonoscopy were normal. Screening for viruses B, and C was negative, and the following laboratory parameters including complete peripheral blood cell count, kidney and liver function tests, and tumor markers (CEA, CA 19-9, AFP) were within normal limits. Liver tumor board concluded to the diagnosis of probable hepatocellular carcinoma with left portal vein TT developed on healthy liver and advised upfront surgery. At laparoscopy, there was no ascites and no suspect lymph node in the liver pedicle. Intraoperative ultrasonography confirmed the tumor characteristics and ruled out additional intra-hepatic lesions. A laparoscopic left hepatectomy was performed. Post-operative course was uneventful, and the patient was discharged home nine days after surgery. Specimen analysis confirmed the resection was R0 and the healthy non-tumor parenchyma. The tumor was composed of atypical spindle cells with a fascicular growth pattern. The proliferation index was estimated at $10 \%$ of tumor cells. Immunohistochemical staining against $\alpha$-smooth muscle actin was strongly positive (Fig. 1B-D). The liver tumor board decided no adjuvant treatment. Eighteen months after surgery, the pa- tient was well without recurrence. The tumor was grade 2 according to the FNCLCC (Fédération Nationale des Centres de Lutte Contre le Cancer). ${ }^{3}$

\section{Case 2}

A sensible right hepatomegaly was discovered in a 53 year-old man complaining of abdominal pain. CT scan showed right liver tumor $(29 \mathrm{~cm}$ in diameter) with compressed but patent portal and hepatic veins. Contrast imaging showed peripheral enhancement at late arterial phase. At MRI, the tumor was hyperintense and hypointense on T2-weighted and T1-weighted images, respectively. Peripheral contrast uptake was seen at arterial phase followed by reinforcement at the portal phase. The right portal vein could not be identified (Fig. 2A, B). Thoracic CT showed 2 infra-centimetric non-specific pulmonary nodules. Percutaneous tumor biopsy at the referring center concluded to intrahepatic cholangiocarcinoma. Upper and lower gastro-intestinal endoscopies as well as liver and kidney function tests, and tumor markers (CEA, CA 19-9, AFP) were normal. Screening for viruses B, C, and HIV was negative. The decision was to perform upfront liver resection with the diagnosis of massive symptomatic intrahepatic cholangiocarcinoma. At laparotomy, there was neither suspicious lymph node nor extrahepatic lesion. Right extended hepatectomy under total vascular exclusion of 

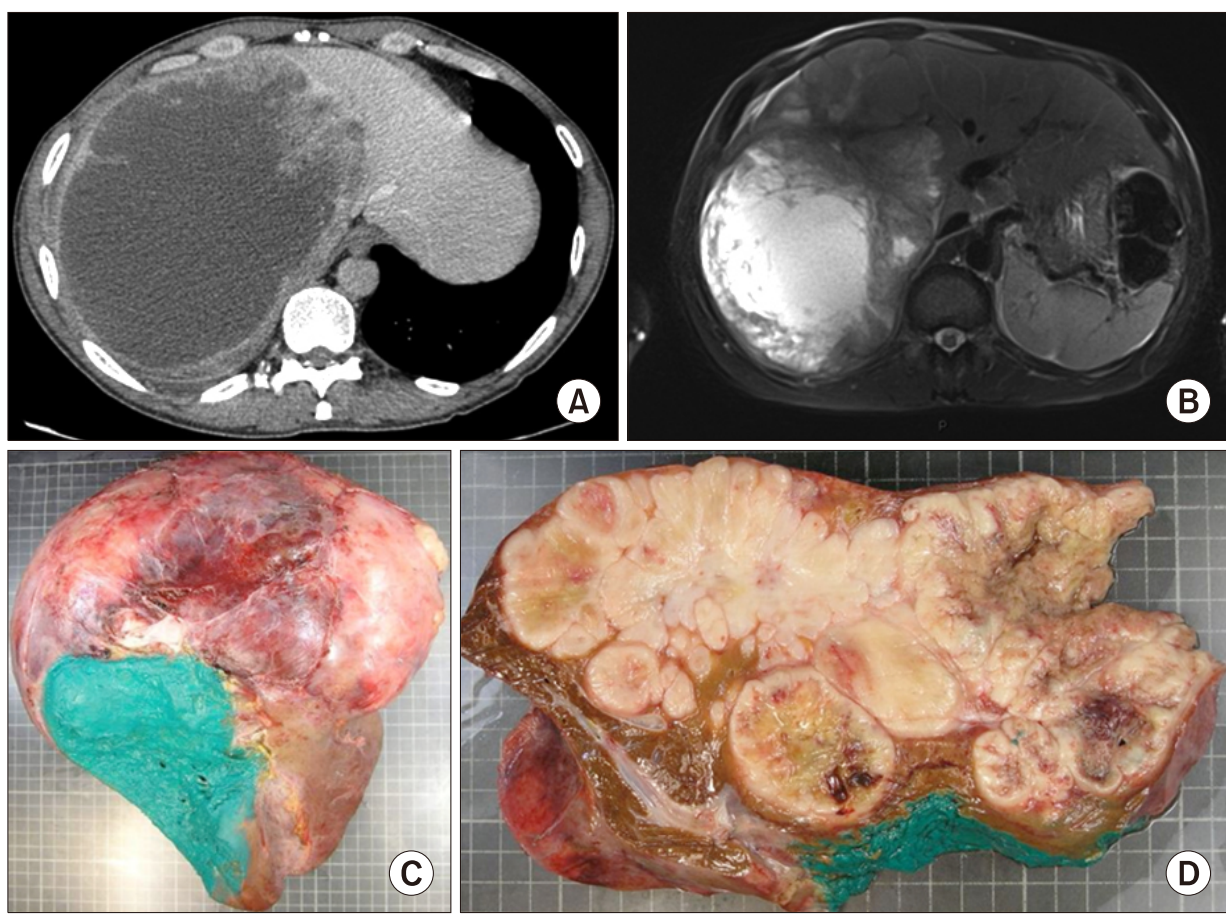

Fig. 2. Giant leiomyosaroma of the right liver. (A) Computed tomography. (B) Magnetic resonance imaging. (C and D) Gross aspects on the resected specimen.

Table 1. Reported cases of leiomyosarcoma of the portal vein and its tributaries in the adult

\begin{tabular}{|c|c|c|c|c|c|}
\hline $\begin{array}{l}\text { 1st author }{ }^{\text {ref }}, \\
\text { year }\end{array}$ & $\begin{array}{c}\text { Sex/age, } \\
\mathrm{yr}\end{array}$ & Localization & Surgical procedure & Margin & Follow-up \\
\hline Wilson $^{19}, 1987$ & $\mathrm{~F} / 28$ & Main portal vein and bifurcation & Operated-not resected & - & Not available \\
\hline Sundaresan $*^{21}, 1990$ & $F / 67$ & Left portal branch & Left hepatectomy & $\mathrm{R} 2$ & Not available \\
\hline Boudjema $^{16}, 2014$ & $\mathrm{~F} / 44$ & Mesenterico-portal junction & $\begin{array}{l}\text { Whipple with portal and biliary } \\
\text { confluence reconstruction }\end{array}$ & R0 & $\begin{array}{l}\text { Recurrence at } 27 \text { months } \\
\text { Died of recurrence at } 47 \\
\text { months }\end{array}$ \\
\hline Gohrbandt $^{20}, 2016$ & $\mathrm{~F} / 71$ & $\begin{array}{l}\text { Main portal vein and right } \\
\text { branch }\end{array}$ & $\begin{array}{l}\text { Right hepatectomy with portal } \\
\text { and biliary reconstruction }\end{array}$ & R0 & $\begin{array}{l}\text { Alive with local recurrence } \\
\text { at } 72 \text { months (personal } \\
\text { communication) }\end{array}$ \\
\hline Gaignard $^{17}, 2019$ & $\mathrm{M} / 53$ & Intrahepatic right portal branch & $\begin{array}{l}\text { Right hepatectomy with portal } \\
\text { and biliary reconstruction }\end{array}$ & R0 & $\begin{array}{l}\text { Alive with no recurrence } \\
\text { at } 6 \text { months }\end{array}$ \\
\hline Present case, 2018 & $\mathrm{M} / 78$ & Intrahepatic right portal branch & Left hepatectomy & R0 & $\begin{array}{l}\text { Alive with no recurrence } \\
\text { at } 18 \text { months }\end{array}$ \\
\hline
\end{tabular}

F, female; M, male

*The portal vein origin was supposed but not formally confirmed since this $30-\mathrm{cm}$ lesion also invaded adjacent structures

the liver with hypothermic portal perfusion and veno-venous bypass was performed ${ }^{4}$ together with lymph node dissection of the liver pedicle. Postoperative course was uneventful, and the patient was discharged home 24 days following surgery. The specimen weighed $5300 \mathrm{~g}$ and contained more than $1 \mathrm{~L}$ of hemorrhagic liquid (Fig. 2C, D). No tumor invasion was found in 5 lymph nodes, and the non-tumor liver parenchyma was normal. The tumor was not encapsulated, and no vascular invasion was found. Immuno-histochemistry showed homogenous and diffuse staining for smooth muscle markers i.e., $\alpha$-smooth muscle actin, h-Caldesmone, and desmin. In addition, the tumor was negative for CD 34, cytokeratin CKAE1/AE3, C-Kit/ CD117, PS100, hepatocyte antigen, and Glypican-3. The proliferation index was estimated at $30 \%$ of tumor cells. The tumor was graded FNCLCC grade 3. Multiple lungs metastases were diagnosed 7 months following surgery. In accordance with the patient, only best supportive care was given, and the patient died with terminal diffuse disease 14 months following surgery. 


\section{Literature search}

A systematic review of the literature by Chi et al. ${ }^{5}$ already identified 109 cases of PHL reported from 1900 to March 2011. To update the latter, our search used the same searching data bases and MeSH terms applied to the period April 2011-April 2019. Reference lists of related articles and review articles were manually screened for additional citations. Our literature search identified 16 additional cases from 12 reports. ${ }^{6-17}$ One registry was not included to obviate redundancy. ${ }^{18}$

\section{DISCUSSION}

Originating from smooth muscle, PHLs can arise from intrahepatic vascular structures, bile ducts or the round ligament. ${ }^{6}$ The presence of a portal vein TT in our first case claims for a portal vein origin of the tumor. Among vascular leiomyosarcomas, those arising from portal vein are very rare and only five cases have been reported. ${ }^{16,17,19-21}$ These cases are summarized in Table 1. R0 resection could be performed in 4 out of 5 evaluable patients. Logically, hepatocellular carcinoma developed in healthy liver with portal vein TT is the first differential diagnosis for this type of PHL. Our first case was treated as so. ${ }^{25}$ The following indirect arguments claim for a biliary origin of the second case: the round ligament and the vessels of the specimen were tumor free.

Diagnostic of PHL needs a high level of suspicion because clinical scenario, ${ }^{6}$ and cross-imaging ${ }^{11,23}$ are not specific. Further, the absence of serological markers often delays diagnosis at the stage of large and/or metastatic tumor. ${ }^{5}$ Concordant with our cases, hepatocellular carcinoma and cholangiocarcinoma are the main differential diagnoses. Indeed, tumor biopsy is the only mean to achieve formal diagnosis of PHL. Intentionally, preoperative tumor biopsy was not performed in our first case because i) cross imaging strongly suggested hepatocellular carcinoma, ii) upfront surgery was indicated for obviously malignant tumor independently of a specific tumor diagnosis, iii) the risks of tumor biopsy (of severe bleeding $=0.6 \%$ to $1.7 \%,,^{24,25}$ of tumor seeding for hepatocellular carcino$\mathrm{ma}=2.7 \%{ }^{26}$ ) was not balanced by the potential benefit of any neoadjuvant treatment. Nevertheless, we acknowledge preoperative biopsy should be considered on a caseby-case basis. Immunohistochemistry easily makes the right diagnostic and staging. ${ }^{3}$ This was not performed at the referral center in our second case and explains the wrong initial diagnosis of cholangiocarcinoma.

Radical R0 hepatectomy is the cornerstone of successful management of PHL. Considering the present update of the systematic review by Chi et al., ${ }^{5} 111$ cases could be analyzed for treatment of which 71 could be operated (64\%). The vast majority of unoperated patients had technically non-resectable disease and/or extra-hepatic metastases. In terms of survival, Chi et al..$^{5}$ could analyze 84 cases. A median overall survival of 19 months (range 0181 months) with 1-, 2-, and 5-year survival rates of $61.2 \%, 41.1 \%$, and $14.5 \%$, respectively was found. Smaller size of lesion and more importantly tumor-free resection margin were identified as independent predictors of improved survival. Specific survival following R0 surgery was not reported. The role of chemotherapy (either for neoadjuvant, adjuvant or for palliative purposes) for PHL, as for leiomyosarcomas in general, is unclear. The role of liver transplantation remains controversial.

In conclusion, leiomyosarcomas of the liver are rarely primitive and preoperative diagnosis needs a high degree of suspicion due to atypical clinical presentation and nonspecific imaging features. Surgery is the mainstay of the management of PHL. R0 resection is the main prognostic factor. The place of perioperative chemotherapy remains debated.

\section{ORCID}

Francesco Esposito: https://orcid.org/0000-0002-8885-4873 Chetana Lim: https://orcid.org/0000-0001-9644-2572

Laurence Baranes: https://orcid.org/0000-0003-2706-4170

Chady Salloum: https://orcid.org/0000-0001-9282-4418

Cyrille Feray: https://orcid.org/0000-0002-3885-2052

Julien Calderaro: https://orcid.org/0000-0002-5370-0807

Daniel Azoulay: https://orcid.org/0000-0002-0932-0540

\section{AUTHOR CONTRIBUTIONS}

Conceptualization: DA.

Data curation: FE, CL, CS, LA, JC, DA.

Formal analysis: FE, CL, LB, CS, CF, JC, DA.

Funding acquisition: Not applicable.

Methodology: FE, CL, DA. 
Project administration: DA.

Visualization: DA.

Writing - original draft: FE, CL, DA.

Writing - review \& editing: DA.

\section{REFERENCES}

1. Liver Cancer Study Group of Japan. Primary liver cancer in Japan. Clinicopathologic features and results of surgical treatment. Ann Surg 1990;211:277-287.

2. Gagnier JJ, Kienle G, Altman DG, Moher D, Sox H, Riley D; CARE Group. The CARE guidelines: consensus-based clinical case report guideline development. J Clin Epidemiol 2014;67: 46-51.

3. Coindre JM. Grading of soft tissue sarcomas: review and update. Arch Pathol Lab Med 2006;130:1448-1453.

4. Azoulay D, Lim C, Salloum C, Andreani P, Maggi U, Bartelmaos $\mathrm{T}$, et al. Complex liver resection using standard total vascular exclusion, venovenous bypass, and in situ hypothermic portal perfusion: an audit of 77 consecutive cases. Ann Surg 2015;262:93104.

5. Chi M, Dudek AZ, Wind KP. Primary hepatic leiomyosarcoma in adults: analysis of prognostic factors. Onkologie 2012;35:210214.

6. Shivathirthan N, Kita J, Iso Y, Hachiya H, Kyunghwa P, Sawada $\mathrm{T}$, et al. Primary hepatic leiomyosarcoma: case report and literature review. World J Gastrointest Oncol 2011;3:148-152.

7. Takehara K, Aoki H, Takehara Y, Yamasaki R, Tanakaya K, Takeuchi H. Primary hepatic leiomyosarcoma with liver metastasis of rectal cancer. World J Gastroenterol 2012;18:5479-5484.

8. Chelimilla H, Badipatla K, Ihimoyan A, Niazi M. A rare occurrence of primary hepatic leiomyosarcoma associated with epstein barr virus infection in an AIDS patient. Case Rep Gastrointest Med 2013;2013:691862.

9. Majumder S, Dedania B, Rezaizadeh H, Joyal T, Einstein M. Tumor rupture as the initial manifestation of primary hepatic leiomyosarcoma. Gastrointest Cancer Res 2014;7:33-34.

10. Lin YH, Lin CC, Concejero AM, Yong CC, Kuo FY, Wang CC. Surgical experience of adult primary hepatic sarcomas. World J Surg Oncol 2015;13:87.

11. Lv WF, Han JK, Cheng DL, Tang WJ, Lu D. Imaging features of primary hepatic leiomyosarcoma: a case report and review of literature. Oncol Lett 2015;9:2256-2260.

12. Hamed MO, Roberts KJ, Merchant W, Lodge JP. Contemporary management and classification of hepatic leiomyosarcoma. HPB
(Oxford) 2015;17:362-367.

13. Iida T, Maeda T, Amari Y, Yurugi T, Tsukamoto Y, Nakajima F. Primary hepatic leiomyosarcoma in a patient with autosomal dominant polycystic kidney disease. CEN Case Rep 2017;6:7478.

14. Giakoustidis D, Giakoustidis A, Goulopoulos T, Arabatzi N, Kainantidis A, Zaraboukas T. Primary gigantic leiomyosarcoma of the liver treated with portal vein embolization and liver resection. Ann Hepatobiliary Pancreat Surg 2017;21:228-231.

15. Zhou F, Huang HZ, Zhou MT, Han SL. Surgical treatment and chemotherapy of adult primary liver sarcoma: experiences from a single hospital in China. Dig Surg 2019;36:46-52.

16. Boudjema K, Sulpice L, Levi Sandri GB, Meunier B. Portal vein leiomyosarcoma, an unusual cause of jaundice. Dig Liver Dis 2014;46:1053-1054.

17. Gaignard E, Bergeat D, Stock N, Robin F, Boudjema K, Sulpice L, et al. Portal vein leiomyosarcoma: a rare case of hepatic hilar tumor with review of the literature. Indian J Cancer 2019;56:8385.

18. Konstantinidis IT, Nota C, Jutric Z, Ituarte P, Chow W, Chu P, et al. Primary liver sarcomas in the modern era: resection or transplantation? J Surg Oncol 2018;117:886-891.

19. Wilson SR, Hine AL. Leiomyosarcoma of the portal vein. AJR Am J Roentgenol 1987;149:183-184.

20. Gohrbandt AE, Hansen T, Ell C, Heinrich SS, Lang H. Portal vein leiomyosarcoma: a case report and review of the literature. BMC Surg 2016;16:60.

21. Sundaresan M, Kelly SB, Benjamin IS, Akosa AB. Primary hepatic vascular leiomyosarcoma of probable portal vein origin. $\mathrm{J}$ Clin Pathol 1990;43:1036.

22. Zhang XP, Wang K, Li N, Zhong CQ, Wei XB, Cheng YQ, et al. Survival benefit of hepatic resection versus transarterial chemoembolization for hepatocellular carcinoma with portal vein tumor thrombus: a systematic review and meta-analysis. BMC Cancer 2017; 17:902.

23. Ferrozzi F, Bova D, Zangrandi A, Garlaschi G. Primary liver leiomyosarcoma: CT appearance. Abdom Imaging 1996;21:157160.

24. Rockey DC, Caldwell SH, Goodman ZD, Nelson RC, Smith AD; American Association for the Study of Liver Diseases. Liver biopsy. Hepatology 2009;49:1017-1044.

25. West J, Card TR. Reduced mortality rates following elective percutaneous liver biopsies. Gastroenterology 2010;139:1230-1237.

26. Silva MA, Hegab B, Hyde C, Guo B, Buckels JA, Mirza DF. Needle track seeding following biopsy of liver lesions in the diagnosis of hepatocellular cancer: a systematic review and metaanalysis. Gut 2008;57:1592-1596. 